\title{
CANCER CÉRVICO UTERINO ESTADIO IIIB TRATADO CON RADIOQUIMIOTERAPIA CONCOMITANTE BASADA EN CISPLATINO: EXPERIENCIA INICIAL HOSPITAL CARLOS VAN BUREN
}

\author{
José Solís C. ${ }^{1,2}$, Mónica Silva V. ${ }^{1}$, Marielisa León P.1,2, Guillermo Merino $H^{1} .{ }^{1}$, Rodrigo Díaz \\ $B .^{1}$, Erasmo Bravo $0^{3,4}$, Cristhian Franck $K^{3,4}$, Javier Egaña $F^{3,4}$ \\ ${ }^{1}$ Servicio de Oncología, Hospital Carlos Van Buren. ${ }^{2}$ Cátedra de Oncología, Escuela de Medicina, Universidad de \\ Valparaíso. ${ }^{3}$ Servicio de Ginecología y Obstetricia, Hospital Carlos Van Buren. ${ }^{4}$ Cátedra de Ginecología y Obstetricia, \\ Escuela de Medicina, Universidad de Valparaíso.
}

\section{RESUMEN}

Objetivo: Analizar retrospectivamente resultados y factores pronósticos de pacientes con cáncer cérvico uterino estadío IIIB tratados con radio quimioterapia concomitante basado en cisplatino. Métodos: Desde septiembre 1999 a diciembre 2002, 48 pacientes con cáncer cérvico uterino estadío IIIB fueron tratados en el Servicio de Oncología del Hospital Carlos Van Buren. La edad promedio fue 46,7 años. La histología correspondió a carcinoma escamocelular en $89,5 \%$ de los casos. El tratamiento consistió en radioterapia pelviana externa y braquiterapia útero-vaginal con baja tasa de dosis (dosis total de 85 Gy a punto $\mathrm{A}$ ). La quimioterapia concomitante consistió en cisplatino semanal $40 \mathrm{mg} / \mathrm{m}^{2}$ con máximo de $70 \mathrm{mg}$, por 5 semanas. Resultados: $85,4 \%$ de las pacientes completó 5 ciclos de quimioterapia. La mediana de sobrevida global fue 39 meses, con una sobrevida global, sobrevida libre de enfermedad y sobrevida libre de recurrencia loco-regional a 3 años de $55,6 \%, 53,3 \%$ y $78,6 \%$, respectivamente. $12,5 \%$ de los pacientes recidivó en pelvis y $22,9 \%$ desarrollaron metástasis a distancia. No se encontraron factores pronósticos de sobrevida global ni sobrevida libre de enfermedad, observando que diámetro tumoral clínico $\geq 7 \mathrm{~cm}$ y no realización de braquiterapia fueron factores de mal pronóstico de sobrevida libre de enfermedad locoregional. Conclusiones: El tratamiento con radioterapia pelviana externa, braquiterapia y quimioterapia concomitante con cisplatino semanal en pacientes con cáncer cérvico uterino estadío IIIB es posible de realizar en el sistema de salud público chileno, con buena tolerancia y resultados comparables a los de la literatura internacional.

\section{PALABRAS CLAVES: Cáncer cérvico uterino localmente avanzado, radio-quimio terapia, cisplatino}

\section{SUMMARY}

Objective: With a retrospective study we propose to analyze results and prognostic factors of patients with FIGO stage IIIB cervical carcinoma of the intact uterine cervix treated with cisplatin-based chemotherapy concurrently with standard radiotherapy. Methods: From September 1999 to December 2002, 48 patients with stage IIIB cervical carcinoma were treated at the department of oncology from Carlos Van Buren Hospital. The mean age was 46.7 years; $89.5 \%$ was squamous cell carcinoma. Treatment consisted of radiotherapy (external-beam radiotherapy and low dose rate brachytherapy) plus weekly cisplatin 
chemotherapy $\left(40 \mathrm{mg} / \mathrm{m}^{2}\right)$ for five weeks. The total dose to point A was 85 Gy. Results: $85.4 \%$ received 5 cycles. The median overall survival was 39 months with 3 year overall survival, disease-free survival and loco-regional disease-free survival of $55.6 \%, 53.3 \%$ and $78.6 \%$, respectively. $12.5 \%$ had recurrence disease in pelvis and $22.9 \%$ developed metastatic disease. We did not find prognostic factors for overall survival and disease-free survival. Prognostic factors related with poor loco-regional disease-free survival were clinical tumor diameter $\geq 7 \mathrm{~cm}$ and patients who did not have brachytherapy. Conclusions: The treatment regimen of external pelvic irradiation, brachytherapy and concurrent weekly cisplatin in patients with stage IIIB cervical carcinomas is feasibility to do in the Chilean public health system, with good tolerance and comparable results to the international trials.

\section{KEY WORDS: Locally advanced cervix cancer, chemo radiation therapy, cisplatin}

\section{INTRODUCCIÓN}

El cáncer cérvico uterino es el segundo cáncer más frecuente en mujeres, siendo una de las principales causas de muerte por cáncer en mujeres de países subdesarrollados, donde la enfermedad frecuentemente se presenta en etapas avanzadas (1-3), afectando a mujeres jóvenes con un promedio de 26 años de vida perdido por cada paciente $(4,5)$. En Chile constituye la primera causa de muerte en mujeres en el grupo entre 20 y 44 años y la sexta en el grupo entre 45 a 64 años (6). La cirugía no es una opción terapéutica para pacientes en estadío IIIB debido a una elevada proporción de márgenes positivos y factores de alto riesgo de recidiva, aumentando la utilización de radioterapia postoperatoria, e incrementando la morbilidad asociada al tratamiento.

La radioterapia ha constituido el tratamiento definitivo estándar para pacientes con cáncer estadío IIIB (7-10). Sin embargo, la dosis de radioterapia necesitada para lograr alta proporción de control tumoral en lesiones de gran tamaño, exceden las dosis toleradas por tejidos normales en la pelvis (11), siendo las recurrencias locales un problema significativo con incidencia de falla pelviana de $35 \%$ a $90 \%$ (7-10). Por lo anterior, se han realizado esfuerzos para mejorar los resultados, que han consistido en estrategias que utilizan 20 más modalidades de tratamiento como son la radioterapia y la quimioterapia.

Basado en los resultados colectivos de 6 estudios randomizados (12-18) y dos meta-análisis $(19,20)$, el tratamiento estándar del cáncer cérvico uterino localmente avanzado es radio quimioterapia concomitante basado en cisplatino. Algunas consideraciones se deben realizar en la aplicación de los resultados de los trabajos randomizados de radio quimioterapia en la población general, ya que estos protocolos no fueron diseñados para encontrar diferencias estadísticas en etapas avan- zadas III y IVA, excluyendo pacientes con ganglios para-aórticos comprometidos.

Luego de la publicación de los primeros 5 estudios prospectivos randomizados (12-17), y con la recomendación de National Cancer Institute de utilizar radio quimioterapia (21) basado en cisplatino en pacientes que requieran radioterapia, el Servicio de Oncología del Hospital Carlos Van Buren adoptó como tratamiento estándar en pacientes con cáncer cérvico uterino localmente avanzado la radio quimioterapia concomitante basada en cisplatino, desde septiembre de 1999.

El propósito de este estudio fue evaluar los resultados de la radio quimioterapia concomitante basada en cisplatino, y la búsqueda de factores pronósticos de sobrevida y control local en pacientes con cáncer cérvico uterino IIIB.

\section{PACIENTES Y MÉTODOS}

Entre septiembre de 1999 a diciembre de 2002, 164 pacientes con el diagnóstico de cáncer cérvico uterino fueron derivados desde hospitales públicos de la IV y $\mathrm{V}$ región al Servicio de Oncología del Hospital Carlos Van Buren. De los 164 pacientes se excluyeron 1 por ser recidiva, 2 por falta de datos en ficha clínica, 4 por ser cáncer de endometrio, 5 fallecidos antes de iniciar tratamiento, 14 por comorbilidad asociada (falla función renal, enfermedad concomitante) y 90 por ser otra etapa de la FIGO. Las fichas clínicas de las 48 pacientes con cáncer cérvico uterino estadío IIIB de la FIGO (22) fueron revisados retrospectivamente. Los pacientes fueron inicialmente evaluados con examen físico y pelviano, hemograma, perfil bioquímico, función renal, radiografía de tórax y pielografía endovenosa. Cistoscopia y enema baritado se utilizaron dependiendo de la sintomatología del paciente. TAC de abdomen y pelvis se obtuvo en la mayoría de los pacientes. Se realizó etapificación en forma conjunta por médicos del 
Servicio de Oncología y del Servicio de Ginecología en reunión multidisciplinaria. Los casos fueron seguidos periódicamente por miembros del servicio de oncología del hospital Carlos Van Buren y médicos del hospital de origen por al menos 3 años o hasta el fallecimiento de la paciente.

La edad promedio fue de 46,7 años (rango: 23 a 70 años), siendo $31,3 \%$ menores de 40 años (Tabla I). El carcinoma escamocelular fue el tipo histológico más común $(89,6 \%)$ seguido del adenocarcinoma (10,4\%). El $83,3 \%$ de los pacientes presentó síntomas o signos al momento del diagnóstico, siendo los más frecuentes: genitorragia $(60,8 \%)$, sinusorragia $(23,9 \%)$, leucorrea $(30,4 \%)$ y dolor pelviano $(15,2 \%)$. El intervalo promedio entre el inicio de los síntomas y el diagnóstico fue de 4,2 meses. El diámetro promedio del cuello uterino fue de $5,3 \mathrm{~cm}$. Se observó compromiso vaginal en $91,7 \%$, llegando a mitad inferior de vagina en el $15,9 \%$.

Se planificó radioterapia externa y braquiterapia intracavitaria y concomitantemente quimioterapia endovenosa semanal basada en cisplatino, según esquemas que se detallan a continuación. Los pacientes recibieron radioterapia pelviana externa utilizando equipo Cobalto-60 o acelerador lineal de $6 \mathrm{MV}, 4$ campos, fracciones de 1,8 a 2 Gy/día, 5 días a la semana por 5 semanas hasta completar dosis de 45 a 50,4 Gy. El campo pelviano APPA se extendió por cefálico hasta unión L4-L5, por caudal $4 \mathrm{~cm}$ distal al margen tumoral y $2 \mathrm{~cm}$ por fuera de pared pelviana en forma lateral. Para los campos laterales los límites céfalo-caudal fueron los mismos que en los campos AP-PA, siendo el límite anterior $1 \mathrm{~cm}$ anterior a la sínfisis pubiana y el posterior incluyo todo el sacro. Una sobreimpresión adicional a parametrios de hasta 10 Gy fue agregada dependiendo de la extensión de la enfermedad, administrándose a través de campos AP-PA paralelos opuestos con un bloque rectangular en línea media.

Se efectuó braquiterapia con baja tasa de dosis en una aplicación intracavitaria, utilizando aplicadores de Fletcher-Suit-Delclos con fuentes de Cesio 137, 1 a 2 semanas luego de completar radioterapia externa. La carga estándar de cesio137 fue de 15-10-10 mgRaEq en el tandem central y $15 \mathrm{mgRaEq}$ en colpostatos vaginales de $2 \mathrm{~cm}$ de diámetro. Se realizó dosimetría computacional de braquiterapia desde el 2001. En caso de no poder realizar braquiterapia se dio sobreimpresión de 61 Gy al tumor central con radioterapia externa. Se planificaron dosis total de 85 Gy a punto A.
Tabla I

\section{CARACTERÍSTICAS DE LAS PACIENTES}

\begin{tabular}{|c|c|}
\hline $\begin{array}{l}\text { Características } \\
\text { Diagnóstico histológico }\end{array}$ & Grupo Total \\
\hline $\begin{array}{l}\text { Carcinoma escamocelular } \\
\text { Adenocarcinoma }\end{array}$ & $\begin{array}{l}89,6 \% \\
10,4 \%\end{array}$ \\
\hline $\begin{array}{l}\text { Edad (años) } \\
\qquad \begin{array}{l}\text { Mediana } \\
\leq 30 \\
31-40 \\
41-50 \\
51-60 \\
60-70\end{array}\end{array}$ & $\begin{array}{c}45 \text { años } \\
6,3 \% \\
29,2 \% \\
33,3 \% \\
14,5 \% \\
16,6 \%\end{array}$ \\
\hline $\begin{array}{l}\text { Diámetro tumoral clínico (cm) } \\
\qquad \begin{array}{l}\text { Mediana } \\
\leq 3 \\
3,1-4 \\
4,1-5 \\
5,1-6 \\
6,1-7 \\
\geq 7\end{array}\end{array}$ & $\begin{array}{c}5,0 \mathrm{~cm} \\
4,2 \% \\
22,9 \% \\
27,0 \% \\
25,0 \% \\
10,4 \% \\
10,4 \%\end{array}$ \\
\hline $\begin{array}{c}\text { Invasión parametrial } \\
\text { Unilateral } \\
\text { Bilateral }\end{array}$ & $\begin{array}{l}33,3 \% \\
66,6 \%\end{array}$ \\
\hline $\begin{array}{l}\text { Invasión vaginal } \\
\text { No } \\
\text { Fondo de saco } \\
\text { Mitad Superior } \\
\text { Mitad Inferior }\end{array}$ & $\begin{array}{c}8,3 \% \\
50 \% \\
27,1 \% \\
14,6 \%\end{array}$ \\
\hline $\begin{array}{l}\text { Clínica } \\
\text { Genitorragia } \\
\text { Sinusorragia } \\
\text { Leucorrea } \\
\text { Dolor }\end{array}$ & $\begin{array}{l}60,9 \% \\
23,9 \% \\
30,4 \% \\
15,2 \%\end{array}$ \\
\hline
\end{tabular}

Se administró quimioterapia concomitante a radioterapia externa consistente en cisplatino 40 $\mathrm{mg} / \mathrm{m}^{2}$ de superficie corporal, con una dosis máxima de $70 \mathrm{mg}$ por ciclo. Se programaron 5 ciclos semanales, comenzando durante la primera semana de radioterapia externa. La quimioterapia fue suspendida transitoriamente cuando el control hematológico semanal mostraba reducción del recuento absoluto de leucocitos menor de $3000 \mathrm{~mm}^{3}$ y/o reducción de neutrofilos menor de $1500 \mathrm{~mm}^{3}$ y/o reducción de plaquetas menor de 100.000 y/o deterioro de función renal manifestada en clearence de creatinina inferior de $50 \mathrm{ml} / \mathrm{min}$ En caso de persistencia de toxicidad grado 3 o mayor por más de 2 semanas la quimioterapia fue suspendida en forma definitiva. Se analizó en forma retrospectiva la toxicidad aguda y crónica producida por radiote- 
rapia utilizando la escala de toxicidad del RTOG/ EORTC (23).

A los pacientes se les registró el tiempo desde el diagnóstico con biopsia hasta la muerte o a la conclusión del estudio en febrero 2005. Las recurrencias fueron calificadas como loco-regionales (pelvis) o a distancia (fuera de la pelvis). Llamamos sobrevida libre de enfermedad loco-regional al tiempo desde el diagnóstico hasta el último control o a la primera recurrencia loco-regional y sobrevida libre de enfermedad también incluyó el primer sitio de metástasis a distancia. Sobrevida global fue determinado como el tiempo desde el diagnóstico hasta la muerte o último control.

Los análisis estadísticos fueron realizados utilizando software estadísticos comerciales, un valor $p<0,05$ fue considerado significativo. Curvas de sobrevida de Kaplan-Meir (24) fueron generadas para analizar sobrevida global, sobrevida libre de enfermedad y sobrevida libre de enfermedad locoregional. El test de Mantel-Cox (25) fue utilizado para realizar análisis univariable de factores pronósticos. Los factores pronósticos estudiados fueron: invasión biparametrial, invasión vaginal 1/3 inferior, tipo histológico, suspensión de quimioterapia, utilización de braquiterapia, edad $\leq 50$ años, hemoglobina menor de $12 \mathrm{~g} / \mathrm{dl}$, días en completar tratamiento y diámetro tumoral $\geq 7 \mathrm{~cm}$.

\section{RESULTADOS}

El promedio de dosis de radioterapia externa sobre pelvis fue de 49,3 Gy, con una mediana de dosis sobre parametrio derecho e izquierdo de 10 y 12 Gy, respectivamente. Se logró realizar braquiterapia en el $85 \%$ de las pacientes, administrando una dosis mediana de $4127 \mathrm{mgRaEg} / \mathrm{hr}$. La mediana de dosis calculada a punto A fue de 80,7 Gy. La mediana de días en completar el tratamiento fue de 62 días. La tolerancia al tratamiento fue buena, completando el curso planeado de tratamiento al $85,4 \%$ de los pacientes (Tabla II), y al menos 4 ciclos al $89,5 \%$.

Al tiempo del análisis en febrero $2005,50 \%$ de las pacientes habían fallecido y $50 \%$ estaban vivos. Con una mediana de seguimiento de 51,5 meses en pacientes vivos (rango: 38 a 66 meses), la mediana de sobrevida fue de 39 meses, con una sobrevida global a 3 años de 55,6\% (Figura 1). El análisis univariable de factores pronósticos de sobrevida global no evidenció factores de mal pronóstico estadísticamente significativos dentro de las variables examinadas (Tabla III).

La sobrevida libre de enfermedad a 3 años fue
Tabla II

TOLERANCIA AL TRATAMIENTO Y CORRELACIÓN CON HEMOGRAMA

\begin{tabular}{lcccc}
\hline Ciclos & $\begin{array}{c}\text { Pacientes } \\
(\%)\end{array}$ & $\begin{array}{c}H B \\
(\mathrm{~g} / \mathrm{dl})\end{array}$ & $\begin{array}{c}\mathrm{GB} \\
\left(\times \mathrm{mm}^{3}\right)\end{array}$ & $\begin{array}{c}\text { Plaquetas } \\
\left(\times \mathrm{mm}^{3}\right)\end{array}$ \\
\hline 1 & 100 & 12,1 & 7388 & 284.177 \\
2 & 93,75 & 12 & 6173 & 266.155 \\
3 & 91,6 & 11,6 & 5071 & 249.891 \\
4 & 89,5 & 11,8 & 4545 & 224.045 \\
5 & 85,4 & 11,6 & 3667 & 208.764 \\
6 & 8,3 & - & - & - \\
\hline
\end{tabular}

HB: hemoglobina. GB: glóbulos blancos.

de $53,3 \%$ (Figura 2), no encontrándose factores pronósticos en el análisis univariable. La sobrevida libre de recurrencia loco-regional a 3 años fue de $78,6 \%$ (Figura 3). El análisis univariable de factores pronósticos mostró que la no realización de braquiterapia $(p=0,016)$ y diámetro tumoral clínico $\geq 7 \mathrm{~cm}(p=0,013)$ fueron factores de mal pronóstico. La sobrevida libre de enfermedad loco-regional a 3 años en el grupo que recibió braquiterapia comparado con el grupo sin braquiterapia fue de $83,4 \%$ y $40 \%$, respectivamente (Figura 4 ). Los pacientes con diámetro tumoral clínico del cervix $\geq 7 \mathrm{~cm}$ tuvieron sobrevida libre de enfermedad loco-regional a 3 años de $66,7 \%$ comparado con

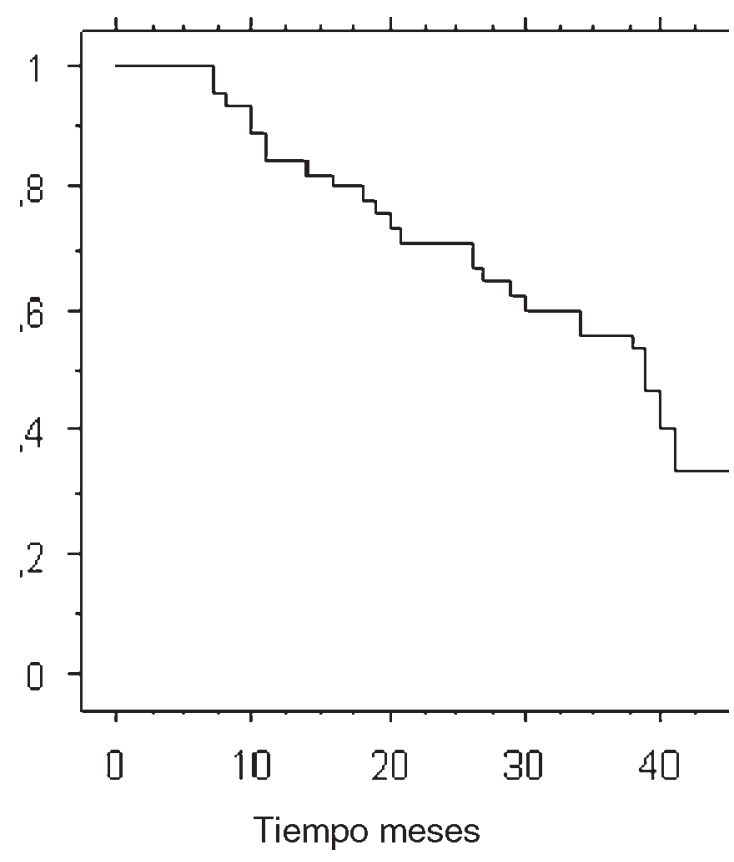

Figura 1. Curva de sobrevida global. 
Tabla III

ANÁLISIS UNIVARIABLE DE FACTORES PRONÓSTICOS

\begin{tabular}{lccc}
\hline Factor pronóstico & $\begin{array}{c}\text { Sobrevida global } \\
\text { Sobrevida libre de } \\
\text { enfermedad }\end{array}$ & $\begin{array}{c}\text { Sobrevida libre de } \\
\text { enfermedad loco-regional }\end{array}$ \\
\hline Diámetro tumoral $\geq 7 \mathrm{~cm}$ & 0,5859 & 0,2731 & 0,0130 \\
Braquiterapia & 0,1852 & 0,1591 & 0,0160 \\
Hemoglobina $<12 \mathrm{~g} / \mathrm{dl}$ & 0,19 & 0,29 & 0,0673 \\
Edad $\leq 50$ años & 0,1828 & 0,2621 & 0,0809 \\
Invasión biparametrial & 0,6131 & 0,5084 & 0,3617 \\
Invasion vaginal 1/3 inferior & 0,4748 & 0,3666 & 0,3578 \\
Tipo histológico & 0,3182 & 0,3203 & 0,2855 \\
Suspensión de quimioterapia & 0,9021 & 0,9107 & 0,7075 \\
Días completar tratamiento & 0,3383 & 0,2127 & 0,1467 \\
\hline
\end{tabular}

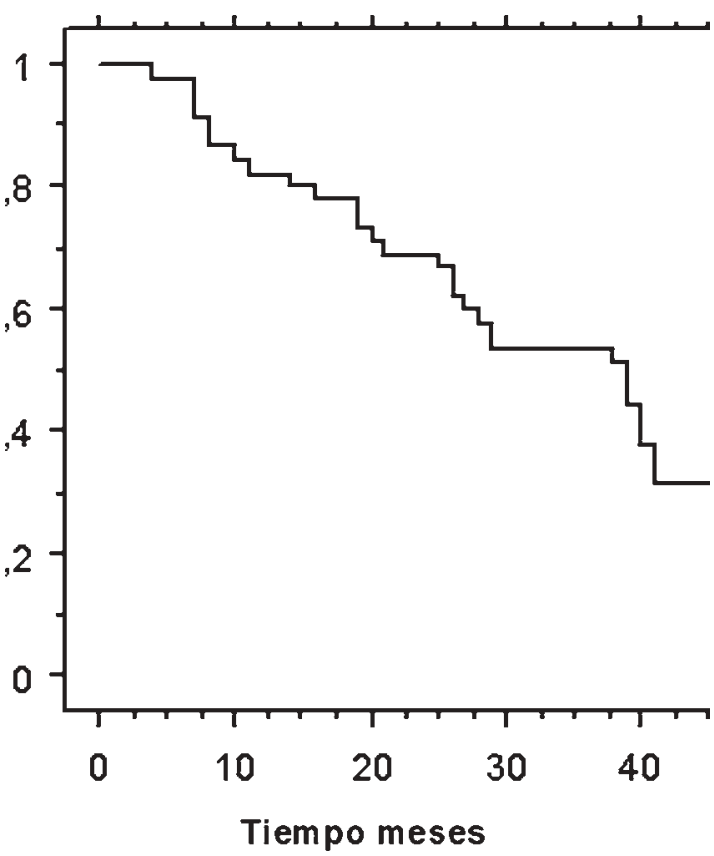

Figura 2. Curva de sobrevida libre de enfermedad.

$82,5 \%$ en pacientes con diámetros tumoral clínico $<7 \mathrm{~cm}$ (Figura 5).

Se observaron recidivas loco-regionales en el $12,5 \%$ de los pacientes y metástasis a distancia en el 22,9\% (Tabla IV). Las recidivas loco regionales fueron exclusivas en 3 casos, concomitantes con metástasis a distancia en 2 casos y 1 caso tuvo recidiva local y luego a distancia. El 66,6\% de las recidivas pelvianas y el $54,54 \%$ de las metástasis a distancia se desarrollaron durante los primeros 24 meses. Los sitios de recurrencias loco-regionales fueron pelvis masiva en 4 pacientes, vagina en 1 paciente y vejiga en 1 paciente.

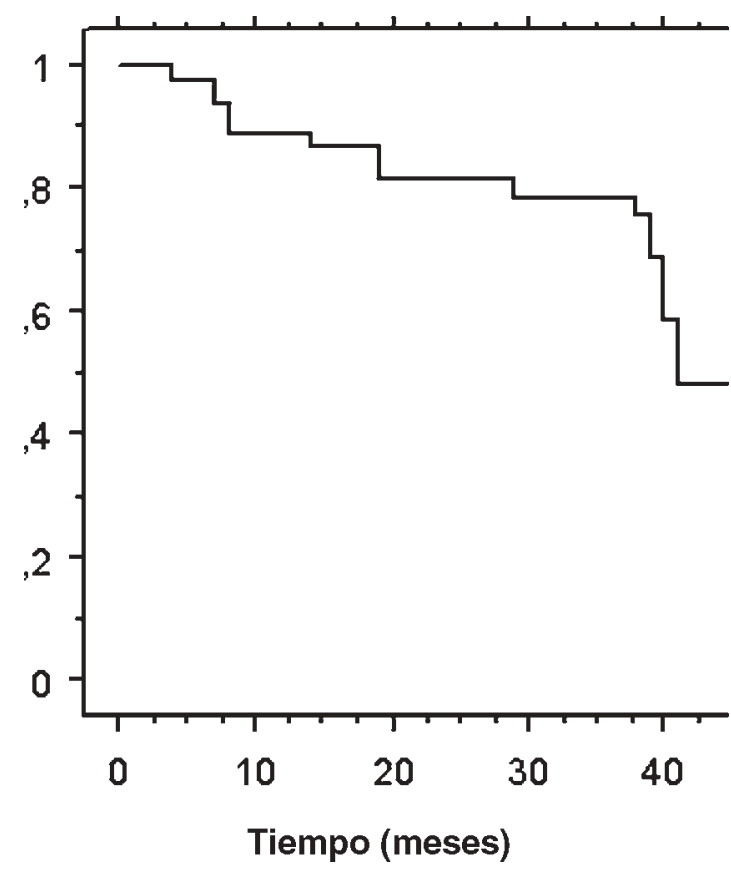

Figura 3. Sobrevida libre de recurrencia loco-regional.

Los sitios de metástasis a distancia fueron ganglios para-aórticos en 4 pacientes, pulmonares en 4 pacientes, hepático 1 paciente y fosa-supraclavicular en 2 pacientes. La mediana de sobrevida desde el diagnóstico de metástasis fue de 4 meses y luego de recidiva local de 5 meses. Se registró 1 caso de segundo cáncer siendo un cáncer renal.

Registramos toxicidad aguda en el $12,5 \%$ de los pacientes. Toxicidad gastrointestinal inferior se observo en el $6,3 \%$, con 1 caso de toxicidad grado 1 y 2 casos de toxicidad grado 2 . Toxicidad hematológica en el 6,3\%, con 2 casos de toxicidad 


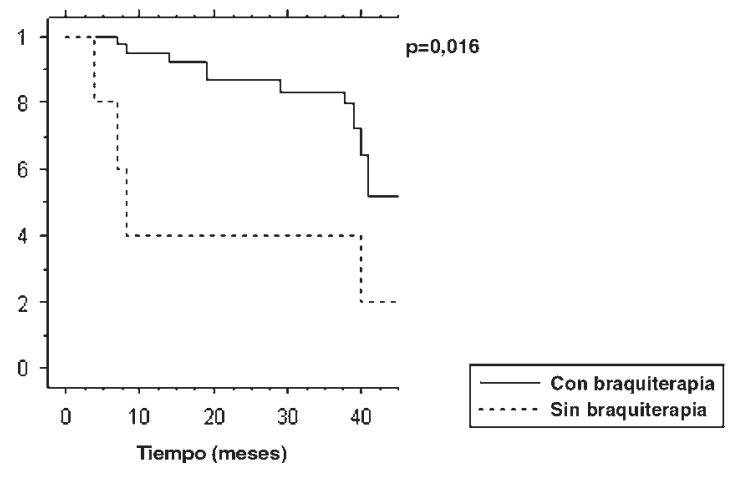

Figura 4. Sobrevida libre de enfermedad loco-regional dependiente de braquiterapia.

grado 2 y 1 caso de toxicidad grado 3 . La mediana de tiempo de producción de toxicidad tardía fue de 32 meses. Se registró toxicidad tardía en el $31,3 \%$ de los pacientes. Toxicidad gastrointestinal se observó en el $20,8 \%$ de los pacientes ( 3 casos grado $1 ; 6$ casos grado 2 y 1 caso grado 4 , fístula recto-vaginal). Toxicidad genito-urinaria se observó en el $10,4 \%$ de los pacientes (grado 1 en 2 casos, grado 2 en 2 casos y grado 3 en 1 caso).

\section{DISCUSIÓN}

El cáncer cérvico uterino invasor es un problema de salud en muchos países subdesarrollados, donde la enfermedad se presenta frecuentemente en etapas avanzadas. Indudablemente los mayores esfuerzos deberían ser dirigidos a mejorar las campañas de screening como el método más efectivo para reducir la mortalidad por cáncer de cérvix,

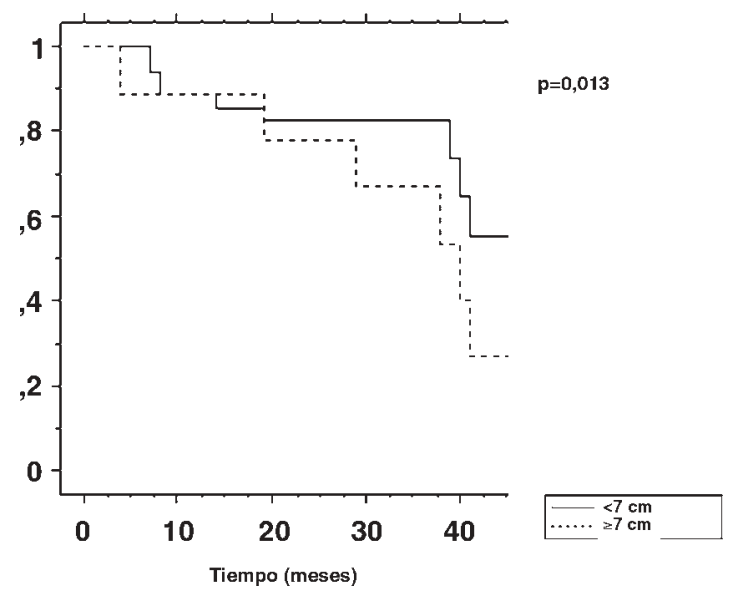

Figura 5. Sobrevida libre de enfermedad loco-regional dependiente de diámetro de cuello uterino. sin embargo, mientras lo anterior sea perfeccionado el uso generalizado de tratamientos más efectivos para cáncer loco-regionalmente avanzados podría contribuir a disminuir la mortalidad.

El promedio de edad $(15,16)$, subtipo histológico (12-16) y características de tratamiento tales como dosis de radioterapia y tiempo de tratamiento $(12,13)$ son similares a los informados por algunos de los trabajos prospectivos randomizados.

La toxicidad aguda fue leve, siendo principalmente gastrointestinal y hematológica transitoria. La tolerancia al tratamiento con un $85 \%$ de los pacientes recibiendo 5 ciclos de quimioterapia fue buena, similar a lo informado en la literatura internacional $(12-14,27,28)$ y corroborando la experiencia nacional publicada por San Martín y cols (26), quienes obtuvieron $95 \%$ de tolerancia a 5 ciclos de quimioterapia, confirmando la factibilidad de realizar este tratamiento en el sistema de salud público chileno. La toxicidad tardía al tratamiento fue principalmente leve a moderada gastrointestinal y genitourinaria, similar a la informada en la literatura $(15,16)$.

Nuestros resultados de sobrevida global con un $55,6 \%$ a 3 años es similar a la informada en otras series internacionales $(15,16,29)$ en estadío IIIB.

Nosotros encontramos que un diámetro tumoral clínico $\geq 7 \mathrm{~cm}$ fue un factor de mal pronóstico de sobrevida libre de enfermedad loco-regional, lo que es un factor pronóstico conocido en pacientes tratadas con radioterapia exclusiva (9,30-33). Aunque impreciso, una evaluación clínica del diámetro tumoral podría reflejar más exactamente el volumen tumoral y por lo tanto predecir más exactamente la extensión de la invasión de la pared pelviana, el cual es un hallazgo altamente subjetivo. Estudios quirúrgicos clásicos demostraron que muchos casos que se pensó por examen físico de

Tabla IV SITIO DE RECURRENCIA

\begin{tabular}{lr}
\hline Sitio de recurrencia & $\%$ \\
\hline Loco-regionales & 12,4 \\
Pelvis masiva & 8,3 \\
Vagina & 2,1 \\
Vejiga & 2,1 \\
Metástasis a distancia & 22,8 \\
Hígado & 2,1 \\
Pulmón & 8,3 \\
Ganglios para-aórticos & 8,3 \\
Supraclavicular & 4,1
\end{tabular}


tener infiltración de pared pelviana fueron fijados a estructuras adyacentes por tejido cicatricial por infecciones antiguas o injurias obstétricas (34). La importancia del diámetro tumoral fue reconocida en la revisión de la FIGO de 1995 (35), produciéndose una división de etapa IB a IB1 en lesiones menores de $4 \mathrm{~cm}$ y IB2 para lesiones $\geq 4 \mathrm{~cm}$. Sin embargo este concepto no se extendió a etapa II - III, siendo una debilidad del sistema de etapificación FIGO-UICC (22), que necesita ser compensado informando los diámetros tumorales clínicos cuando los resultados se reportan por etapas permitiendo comparación entre publicaciones. Estos resultados enfatizan el valor de un cuidadoso examen pelviano para evaluar la extensión pelviana y el volumen de la enfermedad central. Aunque no se encuentran en la clasificación de la FIGO, imágenes modernas incluyendo ecografía transvaginal, tomografía computarizada y resonancia nuclear magnética proveen una determinación más confiable del diámetro tumoral, pudiendo ser usado prospectivamente en todos los casos para calcular medidas y correlacionarlo con resultados.

Otro factor pronóstico de sobrevida libre de enfermedad loco-regional encontrado en nuestra casuística fue el uso de braquiterapia, lo que también es un factor pronóstico reconocido en trabajos de radioterapia exclusiva $(30,36)$, debiendo realizar el máximo esfuerzo para su utilización.

Es importante que continúen las investigaciones en los campos de imágenes como el FDG$\mathrm{PET}$, el rol de los diferentes esquemas de quimioterapia (38-41) y nuevas técnicas de radioterapia (42-46) para mejorar los resultados.

Nuestra experiencia demuestra que el tratamiento con radioterapia pelviana externa, braquiterapia y quimioterapia concomitante basada en cisplatino semanal $\left(40 \mathrm{mg} / \mathrm{m}^{2}\right)$ en pacientes con cáncer cérvico uterino estadío IIIB es posible de realizar en el sistema de salud público chileno, con buena tolerancia y resultados comparables a los de la literatura internacional.

Agradecimientos: Al Dr Carlos Pérez, Departamento Radioterapia Oncológica, Mallinckrodt Institute of Radiology, Washington University, Saint Louis, MO, por haber revisado críticamente el manuscrito.

\section{BIBLIOGRAFÍA}

1. Estimates of the worldwide mortality from 25 cancers in 1990. Pisani P, Parkin DM, Bray F, Ferlay J. Int J Cancer 1999;83:8-29.
2. Global Cancer Statistics. Parkin DM, Pisani P, Ferlay J. CA Cancers Clin 1999;49:33-64.

3. Stat Bite: Cervical Cancer Mortality Worldwide. J Nat Can Inst 2006;98(7): 434.

4. SEER Cancer Statistics Review, 1973-1996, NCl, 1999.

5. Stat Bite: Average years of life lost from cancer. J Nat Can Inst 2001; 93 (5):341.

6. Ministerio de Salud de Chile, Programa Nacional de Cáncer. www.minsal.cl

7. Barrillot I, Horiot JC, Pigneux J, Schraub S, Pourquier H, Daly N, et al. Carcinoma of the intac uterine cervix treated with radiotherapy alone: A French Cooperative Study: Update and multivariate analysis of prognostic factors. Int J Radiation Oncology Biol Phys 1997;38(5):969-78.

8. Perez CA, Grigsby P, Nene S, Camel HM, Galakatos A, Kao M-S. Effect of Tumor size on the prognosis of carcinoma of the uterine cervix treated with irradiation alone. Cancer 1992;69:2796-806.

9. Perez CA, Grigsby P, Chao KS, Mutch D, Lockett MA. Tumor size irradiation dose and long-term outcome of carcinoma of uterine cervix. Int $\mathrm{J}$ radiation Oncology Biol Phys 1998;41(2):307-17.

10. Lanciano RM, Won M, Coia LR, Hanks GE. Pretreatment and treatment factors associated with improved outcome in squamous cell carcinoma of the uterine cervix: a final report of the 1973 and 1978 patterns of care studies. Int J Radiation Oncology Biol Phys 1991;20:667-76.

11. Fletcher GH. Clinical dose-response curves of human malignant epithelial tumours. $\mathrm{Br} \mathrm{J}$ Radiol 1973;46:1-12.

12. Rose P, Bundy B, Watkins E, Thig-Pen T, Deppe G, Maiman $\mathrm{M}$, et al. Concurrent cisplatin-based radiotherapy and chemotherapy for locally advanced cervical cancer. N Eng J Med 1999;340(15):1144-53.

13. Whitney C, Sause W, Bundy B, Malfetano J, Hannigan E, Fowler W, et al. Randomized comparison of fluorouracil plus cisplatin versus hydroxyurea as an adjuvant to radiation therapy in stage IIB-IVA carcinoma of the cervix with negative para-aortic lymph nodes: A Gynecologic Oncology Group and Southwest Oncology Group Study. J Clin Oncol 1999;17(5):1339-48.

14. Keys H, Bundy B, Stehman F, Muderspach L, Chafe W, Suggis III C, et al. Cisplatin, radiation and adjuvant hysterectomy compared with radiation and adjuvant hysterectomy for bulky stage IB cervical carcinoma. N Eng J Med 1999;340(15):1154-61.

15. Eifel P, Winter K, Morris M, Levenback C, Grigsby $P$, Cooper J, Rotman M, Gershenson D, Mutch D. Pelvic irradiation with concurrent chemotherapy versus pelvic and para-aortic irradiation for high-risk cervical cancer: an update of Radiation Therapy Oncology Group Trial (RTOG) 90-01. J Clin Oncol 2004;22(5):872-80.

16. Morris M, Eifel P, Lu J, Grigsby P, Levenback C, Stevens $\mathrm{R}$, et al. Pelvic Radiation with concurrent 
chemotherapy compared with pelvic and para-aortic radiation for high-risk cervical cancer. $\mathrm{N}$ Eng $\mathrm{J}$ Med 1999;340:1137-43.

17. Peters W, Liu P, Barret R, Stock R, Monk B, Berek $\mathrm{J}$, et al. Concurrent chemotherapy and pelvic radiation therapy compared with pelvic radiation therapy alone as adjuvant therapy after radical surgery in high risk early stage cancer of the cervix. J Clin Oncol 2000;18(8):1606-13.

18. Pearcey R, Brundage M, Drovin P, Jeffrey J, Lukka $\mathrm{H}$, Maclean $\mathrm{G}$, et al. Phase III trial comparing radical radiotherapy with and without cisplatin chemotherapy in patients with advanced squamous cell cancer of the cervix. J Clin Oncol 2002;20(4);966-72.

19. Green J, Kirwan J, Tierney J, Symonds P, Collingwood M, Williams C. Survival and recurrence after concomitant chemotherapy and radiotherapy for cancer of the uterine cervix: a systematic review and meta-analysis. Lancet 2001;358:781-6.

20. Lukka $H$, Hirte $H$, Fyles A, Thomas $G$, Elit L, Johnston $\mathrm{M}$, et al. Concurrent cisplatin-based chemotherapy plus radiotherapy for cervical cancer: a meta-analysis. Clinical Oncology 2002;14:203-12.

21. Clinical Announcement NCl. US. Department of Health and Human Services, Public Health Service. National Institutes of Health, 1999.

22. American Joint Committee on Cancer. AJCC: Cancer Staging Manual, Sixth Edition: Editorial Springer 2002; 267-273.

23. Cox JD, Stetz J, Pajaj T. Toxicity criteria of the radiation Therapy Oncology Group (RTOG) and the European Organization for research and treatment of cancer (EORTC). Int J Radiation Oncology Biol And Phys 1995;31:1341-6.

24. Kaplan EL, Meir P. Nonparametric estimation for incomplete observations. J Am Stat Assoc 1958;53: 457-81.

25. Mantel N, Haenzel W. Chi-square test with onedegree of freedom extensions of Mantel-Haenzel procedure. J Am Stat Assoc 1963;58:690-700.

26. San Martin R, Suarez E, Olfos P, Escudero B, Rojas $\mathrm{L}$, Larraguibel $\mathrm{P}$, et al. Cáncer cervicouterino en estadío IB2: Tratamiento con radioquimioterapia concomitante y cirugía. Rev ChIL OBstet GineCOL 2003; 68(6):447-57.

27. Lanciano R, Calkins A, Bundy B, Parham G, Luci III $\mathrm{J}$, Moore D, et al. Randomized comparison of weekly cisplatin or protracted venous infusión of fluoracil in combination with pelvic radiation in advanced cervix cancer: A Ginecologic Oncology Group Study. J Clin Oncol 2005;23:8289-95.

28. Kirwan J, Symonds P, Green J, Tierney J, Collingwood M, Williams $\mathrm{C}$. A systematic review of acute and late toxicity of concomitant chemoradiation for cervical cancer. Radiotherapy and Oncology 2003;68:217-26.

29. Cetina L, Rivera L, Hinojosa J, Poitevin A, Uribe J, Lopez-Graniel C, et al. Routine management of locally advanced cervical cancer with concurrent radiation and cisplatin. Five-year results. BMC Women's Health 2006,6:3 (Published online 2006 February 7. doi: 10.1186/1472-6874-6-3).

30. Lanciano RM, Martz K, Coia LR, et al. Tumor and treatment factors improving outcome in stage IIIB cervix cancer. Int J Radiat Oncol Biol Phys 1991;20: 95-100.

31. Kovalic JL, Perez CA, Grigsby PW, Lockett MA. The effect of volume of disease in patients with carcinoma of the uterine cervix. Int $\mathrm{J}$ Radiat Oncol Biol Physics 1991;21:905-10.

32. Stehman FB, Bundy BN, DiSaia PJ, Keys HM, Larson JE, Fowler WC. Carcinoma of the cervix treated with radiation therapy: I. A multivariate analysis of prognostic variables in the Gynecologic Oncology Group. Cancer 1991;67:2776-85.

33. Eifel PJ, Morris M, Wharton JT, Oswald MJ. The influence of tumor size and morphology on the outcome of patients with FIGO stage IB squamous cell carcinoma of the uterine cervix. Int $\mathrm{J}$ Radiat Oncol Biol Physics 1994;29:9-16.

34. League of Nations Health Organization inquiry into the results of radiotherapy in cancer of the uterus. Atlas illustrating the division of cancer of the uterine cervix into four stages according to the anatomoclinical extent of growth. Stockholm: KunglBoktryckeriet P.A. Norstedt \& Sener;1938.

35. Modifications in the staging for stage I vulvar and stage I cervical cancer. Report of The FIGO Committee on Gynecologic Oncology. International Federation of Gynecology and Obstetrics. Int. J Gynaecol Obst. 1995; 50 (2): 215-6

36. Logsdon M, Eifel P. FIGO III B squamous cell carcinoma of the cervix: An analysis of prognostic factors emphasizing the balance between external beam and intracavitary radiation therapy. Int $\mathrm{J}$ Radiat Oncol Biol Phys 1999; 43(4):763-75.

37. Saibishkumar E, Patel F, Sharma C, Karunanidhi G, Ghoshal S. Prognostic value of response to external radiation in stage IIIB cancer cervix in predicting clinical outcomes: A retrospective analysis of 556 patients from India. Radiotherapy and Oncology 2006;79:142-6.

38. Higgins R, Naumann W, Hall J, Haake M. Concurrent carboplatin with pelvic radiation therapy in the primary treatment of cervix cancer. Gynecol Oncol 2003;89:499-503.

39. Dubay R, Rose P, O'Malley D, Shalodi A, Ludin A, Selim M. Evaluation of concurrent and adjuvant carboplatin with radiation therapy for locally advanced cervical cancer. Gynecol Oncol 2004;94(1):1214.

40. Lorvidhaya V, Chitapanarux I, Sangruchi S, Lertsanguansinchai $\mathrm{P}$, Kongthanarat $\mathrm{Y}$, Tangkaratt $\mathrm{S}$, et al. Concurrent mitomycin C, 5-fluoracil and radiotherapy in the treatment of locally advanced carcinoma of the cervix: a randomized trial. Int J Radiat Oncol Biol Phys 2003;55(5):1226-32.

41. Long III H, Bundy B, Grendys E, Benda J, McMeekin 
S, Sorosky J, et al. Randomized phase III trial of cisplatin with or without topotecan in carcinoma of the uterine cervix: A Gynecologic Oncology Group Study. J Clin Oncol 2005;23:4626-33.

42. Mundt AJ, Roeske JC, Ljan AE, et al. Initial clinical experience with intensity-modulated whole pelvis radiation therapy in women with gynecologic malignancies. Gynecol Oncol 2001;82:456-63.

43. Portelance L, Chao KS, Grigsby PW, Bennet H, Low D. Intensity-modulated radiation therapy (IMRT) reduces small bowel, rectum and bladder doses in patients with cervical cancer receiving pelvic and para-aortic irradiation. Int $\mathrm{J}$ Radiation Oncol Biol Phys 2001;51:261-6.

44. Mundt AJ, Lujan AE, Rotmensh J, et al. Intensity modulated whole pelvic radiotherapy in women with gynecologic malignancies. Int $\mathrm{J}$ Radiat Oncol Biol Phys 2002;52:1330-7.

45. Brixey CJ, Roeske JC, Lujan AE, et al. Impact of Intensity modulated radiotherapy on acute hematologic toxicity in women with gynecologic malignancies. Int J Radiat Oncol Biol Phys 2002;54:138896.

46. Gertzen K, Colonello K, Heron DE, Lalonde RJ, Fitian ID, Comerci JT, Selvaraj RN, Varlotto JM. Feasibility of concurrent cisplatin and extended field radiation therapy (EFRT) using intensity-modulated radiotherapy (IMRT) for carcinoma of the cervix. Gynecologic Oncology 2006;102(2):182-8. Epub 2006; Mar 3. 